\title{
Navigating Conferences to Enhance Science Diplomacy and Communication in the Post-COVID-19 Era
}

Laura Borth $^{1}$, Rachel Gilfarb ${ }^{2}$, Alex Hsain ${ }^{3}$, Christopher T. Jackson $^{4}$, Annabelle T. Lolinco ${ }^{5,10}$, Laetitia Meyrueix ${ }^{6}$, Sindhu S. Nathan ${ }^{7}$, Meredith N. Schmehl ${ }^{8,10}$, Pawan Upadhyay ${ }^{9}$

Affiliations:

1. Department of Nutritional Sciences, University of Wisconsin-Madison, Madison, WI

2. Department of Psychology, The Ohio State University, Columbus, $\mathrm{OH}$

3. Department of Materials Science and Engineering, NC State University, Raleigh, NC

4. Department of Chemistry, University of California, Berkeley, Berkeley, CA

5. Department of Chemistry, Iowa State University, Ames, IA

6. Department of Nutrition, University of North Carolina at Chapel Hill, Chapel Hill, NC

7. Department of Chemical Engineering, Stanford University, Stanford, CA

8. Department of Neurobiology, Duke University, Durham, NC

9. Washington University School of Medicine, St. Louis, MO

10. National Science Policy Network

Corresponding Author: MNS (hello@meredithschmehl.com)

\section{Introduction}

COVID-19 has renewed the importance of science diplomacy, with international cooperation and scientific collaboration across borders more critical than ever to combat the pandemic. To better disseminate rapidly developing innovations and scientific expertise, there is a need to reevaluate the relationship between modes of scientific communications and traditional diplomatic channels, particularly as both are buoyed by drastic global increases in internet use. ${ }^{1,2}$ At the same time, the ease by which information spreads also permits dangerous pitfalls scientists must avoid; COVID19 brought an "infodemic" of misinformation dominated by fake medical advice, unproven treatments, and conspiracy theories. ${ }^{3}$ Science diplomacy represents an important avenue to address the infodemic on domestic and international issues by building relationships and trust among key leaders. ${ }^{4}$

Although science diplomacy is traditionally between national governments or international organizations such as the United Nations, individual connections between scientists from different countries is itself a form of science diplomacy. ${ }^{5}$ Scientific conferences are a key component of science diplomacy and more broadly bring scientists and their industrial, governmental, and philanthropic partners together from across the world to discuss major scientific advances and challenges. ${ }^{6}$

However, in the midst of a global pandemic, traveling and congregating with a large group of people for a traditional conference is no longer viable. ${ }^{7}$ While some multinational conferences, such as COP26, have been postponed, scientific conferences have adapted their format even before COVID-19. For example, before the pandemic halted travel, the American Geophysical Union 
opted for a virtual-only 2020 conference to reduce $\mathrm{CO}_{2}$ emissions, a strategy that will become increasingly necessary as we tackle climate change.

Beyond reducing carbon footprints, virtual conferences increase accessibility by reducing financial and regulatory barriers. The average cost of attending an international conference can range from 776 to 1012 euros (918 to 1198 USD), which can be unaffordable for early-career researchers (ECRs). ${ }^{8}$ Additionally, visa restrictions severely limit opportunities for scientists from Islamic countries, for example. ${ }^{5}$ Virtual formats accommodate translation into many languages and avoid physical barriers for those with disabilities. Virtual conferences are then more accessible to traditionally excluded voices, allowing those from underrepresented countries and groups, including racial and ethnic minorities, disabled scientists, and economically-disadvantaged communities to participate in science and science diplomacy conversations. This increased diversity of thought can improve science diplomacy, as interactions with underrepresented groups are less likely to organically occur in-person due to social barriers.

The COVID-19 pandemic presents an opportunity to permanently transform scientific conferences to improve international scientific communication and diplomacy. Because scientific conferences are integral to network-building, switching to a virtual platform may have an immense influence on how scientists communicate with international peers, government leaders, the public, and industry partners, and conduct diplomatic relations.

However, while conference virtualization addresses some COVID-19 challenges, others still remain. Virtual conferences can hinder science diplomacy, as difficult conversations or agreements are challenging when in-person cues such as body language are lost. Additionally, accessibility obstacles such as unstable internet connection, lack of video conferencing equipment, time zone differences, dueling personal commitments, accommodations for individuals with disabilities, and limited human-to-human and spontaneous connections are just some of the many challenges that must be addressed. ${ }^{9}$

Scientific conferences are critical for elevating voices outside of traditional leadership roles in science diplomacy. Through international scientific collaborations that will form during such conferences, the scientific community can broker new partnerships where formal political relationships are strained. ${ }^{5}$ Thus, it is essential that virtual scientific conferences are made accessible in order to facilitate effective science diplomacy. Here we explore the major obstacles of in-person conferences (high cost, locally-focused, and less equitable) which limit how global conversations can benefit from scientific knowledge, as well as opportunities offered by virtual meetings (affordability, global participation, equitable nature) to foster science diplomacy. Further, we provide recommendations that can enable future conferences, both fully or partially virtual, to strengthen science diplomacy post-COVID-19.

\section{Conference Considerations}

\section{Attendance}

Attending scientific conferences and presenting research to an international audience of peers is a critical professional opportunity for ECRs. Due to the pandemic, many conferences have been 
forced to cancel ${ }^{10}$ or go entirely virtual, ${ }^{11}$ potentially affecting the career prospects of ECRs across multiple fields. Conferences focused on science diplomacy have also been postponed, ${ }^{12}$ hindering international relationships and agreements. The pandemic has enabled the virtual nature of conferences to be more prominent; however, only time will tell whether this change is long lasting. Regardless, the virtualization of conferences highlights key barriers and opportunities to improve participation, especially with regard to accessibility.

Beyond typical attendees, conferences must engage a broader audience to foster stronger scientific and diplomatic ties. ECRs can be excluded from traditional conferences due to significant up-front costs with delayed or no reimbursement. ${ }^{13}$ Attendee bias is further exacerbated when costs increase for international travel. ${ }^{14}$ Virtual conferences offer an opportunity to significantly decrease ${ }^{15,16}$ or remove costs, ${ }^{11}$ allowing expanded participation. ${ }^{14}$ This larger audience may lead to a greater diversity of participants, including younger scientists (e.g., undergraduates), casual science enthusiasts, teachers, journalists, and scientists from different geographic regions, ${ }^{14,17}$ and can foster more inclusive international science relationships.

However, virtual conferences also have inherent drawbacks that can impede diplomatic progress. One prerequisite for virtual conference participation is reliable internet access for several hours a day, across multiple days. ${ }^{14,17}$ While internet usage has increased, it is not equal across the world, ${ }^{18}$ and unreliable access may cause difficulties in live discussions or recorded media. Another barrier is accommodating the time zones of all participants, ${ }^{17}$ as some participants must log in at atypical hours to participate. Thus, some attendees may lose opportunities to form meaningful international connections, because those opportunities are only available during typical sleeping hours. Although some attendees may choose to sacrifice sleep to participate, others may face constraints in doing so because of other commitments like childcare. This time zone barrier does not arise in the traditional, in-person conference format.

\section{Talks and Q\&A}

Oral conference talks are a critical opportunity to share strategies to address global problems and help ECRs establish themselves as subject matter experts on issues relevant to science diplomacy. By going virtual, traditionally small conferences can increase attendance, allowing talks to reach wider audiences. Time zone barriers can be mitigated by allowing recorded sessions to be viewed and commented upon asynchronously. This expanded network of conference participants can increase the breadth of the questions asked and inspire diverse new scientific ideas.

In addition to offering many of the career benefits of traditional conferences, virtual conferences can also make science more equitable for both speakers and attendees by giving more access to underrepresented voices. Traditional conferences may reinforce inequities by insulating wellconnected and well-funded researchers and perpetuating a narrow view of who is qualified to share their work. As fewer social and financial resources are required to attend virtual conferences, a more diverse pool of applicants can present. Virtual talks expand the reach of lesser known research, especially from smaller and less-funded research groups, which can bring new solutions to the forefront of international attention. Additionally, by including body language in videos, live captioning, and transcripts and recordings after the conference, information can be made more accessible to a global audience. ${ }^{19}$ However, this access must be carefully moderated to ensure data 
privacy. Establishing a recording policy or confidentiality agreement, or only allowing video access to registered conference attendees could address these concerns. ${ }^{20}$

During and after the talk, virtual settings can improve question and answer (Q\&A) sessions typical of scientific conferences. Benefits of virtual Q\&As include enhanced moderator control, prioritization of the most meaningful questions, community-sourced answers and engagement throughout the talk, and equal opportunity to participate. ${ }^{21}$ The live chat, unique to the virtual space, can allow live questioning during the presentation, engaging attendees with the speaker and one another in real time. ${ }^{22}$ In one instance, conference organizers found that private messages between speakers and moderators allowed for a more relevant and accessible Q\&A compared to an in-person event. ${ }^{22}$ Surveys indicate that chats also give participants a voice more frequently than would be possible at a live meeting. ${ }^{21}$ Post-COVID-19, the continued use of online Q\&A sessions could create an inclusive space that amplifies previously neglected voices and makes scientific research more accessible for science diplomacy.

\section{Networking}

A more challenging hurdle in virtual conferences is imitating the informal interactions, often over food and drinks, that make conferences valuable networking opportunities. These conversations are an avenue for scientists to develop ideas and build relationships that form the basis of science diplomacy. We need platforms that emulate this environment to foster diplomatic relations between scientists, government and intergovernmental entities, and industrial and community partners. Surveys have shown that regardless of an in-person or virtual setting, conference attendees most value the ability to interact with a variety of professionals, a comfortable environment, and the ability to jump into existing conversations. ${ }^{23}$ Many virtual networking platforms are able to provide participants these desired features, making these platforms an increasingly viable option to replicate in-person conference networking.

Two examples of many such platforms are High Fidelity and Mozilla Hubs. High Fidelity ${ }^{24}$ allows conference organizers to choose a virtual "environment," such as a bar or a banquet hall, for participants to navigate. Participants are assigned an avatar that can move throughout the environment and converse with their neighbors via a microphone. The platform mimics in-person interactions by adjusting the audio based on the position of the avatar, amplifying the voices of the nearby avatars, and dampening voices farther away. Platforms like High Fidelity allow scientists to share ideas in small groups, rather than individually as in teleconferencing software. Similarly, Mozilla $\mathrm{Hubs}^{25}$ and analogous services enable participants to interact informally while watching a synchronous event such as a lecture. ${ }^{26}$ If used in a networking capacity, Mozilla Hubs users can upload 3D models, videos, or other media to use in conversation.

Virtual networking platforms can be a double-edged sword for accessibility. These platforms enable communication tools such as screen readers and text-to-speech systems to improve nonverbal conversation. However, technical limitations such as a threshold for the number of participants per room or increased internet bandwidth requirements may limit both the quantity and quality of interactions and constrain opportunities to foster diplomatic ties. The choice of networking platform is critical to ensure accessibility and ease of diplomatic conversations at virtual conferences. 


\section{Public Engagement}

According to 3M's State of Science Index, trust and interest in science have increased in $2020 .{ }^{27}$ Because of streaming, video conferencing, and instant messaging platforms, scientists and the public can engage in new ways. These interactions do not need to cease in a post-pandemic world, as conferences provide a unique opportunity to enhance public engagement with science. ${ }^{28,29}$ While some in-person conference elements may resume post-pandemic, retaining elements of the virtual format can help maximize public engagement. This engagement can be a powerful way to generate momentum and political incentive for science diplomacy solutions.

In-person and virtual conferences provide distinct opportunities and barriers to public engagement. Virtual lectures can be streamed or recorded, allowing improved engagement with those who cannot attend synchronously and providing an accessible and low commitment avenue to engage. ${ }^{14,30}$ Traditionally, in-person "mutual learning" sessions, where scientists and the public engage as peers to improve applicability and direction of research, can be a platform for scientists and non-scientists to interact and identify real-world applications for the research.$^{28}$ However, virtual roundtables may not facilitate this kind of interaction due to the logistical difficulties of hosting large groups, in addition to the nonverbal cues that may be missed online. A hybrid model of both in-person and virtual conferences can counterbalance barriers to public engagement associated with each approach. ${ }^{31}$ Blended models would improve affordability, inclusivity, and information accessibility to stimulate public discourse in science and policy.

\section{Recommendations}

As COVID-19 forced many conferences into virtual spheres, the benefits and pitfalls of online formats became apparent. Hybrid-model conferences, with both virtual and in-person sessions, offer the benefits of both such as broadening accessibility and decreasing contribution to $\mathrm{CO}_{2}$ emissions. With this hybrid model, scientific conferences could be more affordable, environmentally friendly, global, and inclusive. These tenets are essential for enhancing science diplomacy in the post-COVID-19 era. We recommend the following best practices for conference organizers considering a hybrid model:

\section{Attendance:}

1. Decrease registration fees. Fees are often a financial barrier; the partially virtual format offers an opportunity to increase accessibility and inclusion by reducing these costs.

2. Raise participation thresholds for online events. While in-person conferences may have a participation limit to prevent crowding, the open nature of virtual conferences can permit additional and diverse attendees to participate. ${ }^{22}$

3. Offer asynchronous events. Multiple instances of the same session and public recordings can make events accessible to a global audience. ${ }^{17}$

Talks and $Q \& A$ :

1. Train moderators and facilitators. Because guiding an effective conversation can be difficult in a virtual format, conference organizers should provide training for virtual moderators. 
2. Use privately moderated and/or interactive chat-based Q\&A. Use of the chat box can give more audience members a voice during virtual Q\&A sessions, especially women, people with different language backgrounds, and individuals with less education. ${ }^{21,32}$ Additionally, private messages between speakers, audience members, and moderators during virtual sessions can prevent one person from monopolizing the speaking time and ensure the questions that most require the speaker's expertise are answered. ${ }^{22}$

3. Encourage interactive or user-led presentations. It can be easy to lose audience attention in a virtual format. To create a comparable interaction in virtual forums, moderators should use chat functions to keep audiences engaged with discussion prompts.

4. Maintain accessibility. Virtual sessions can be made more accessible by incorporating captioning in multiple languages during a talk, whether broadcasted or on demand. ${ }^{19}$

5. Include asynchronous $\mathbf{Q} \& \mathbf{A}$ features. International speakers and audience members benefit from asynchronous discussion as time allows. ${ }^{22}$

Networking:

1. Use software to mimic in-person interactions. Software such as High Fidelity can simulate in-person interactions with multiple speakers. The remote format ensures more people can engage in networking opportunities.

2. During presentations, create informal, randomized pods for discussion. Software such as Mozilla Hubs can help attendees engage with the presentation material, offering a route to scientific partnerships that transcend institutional or national borders.

3. Ensure all stakeholders are distributed throughout networking platforms. Equal distribution of stakeholders from a variety of backgrounds, including industry, academia, and policy, is critical to the development of partnerships to improve transnational scientific cooperation.

\section{Public Engagement:}

1. Livestream and record events. Through virtual sessions, organizers can take full advantage of live streams or recordings to distribute the material to wider audiences. ${ }^{14,30}$ Additionally, those unable to attend the event in person would benefit from access to recordings.

2. Organize "mutual learning" sessions. Conferences can encourage public engagement by organizing sessions where scientists and non-scientists discuss as peers or learn about a topic together. These events can improve applicability and direction of research and foster public appreciation of science. ${ }^{28}$

\section{Conclusion}

We present opportunities and barriers to scientific communication uncovered by the COVID-19 pandemic, and recommend approaches to strengthen the communication interface for science diplomacy. Conferences remain an important tool for science diplomacy and communication, providing an environment for networking, scientific collaboration, and international partnerships. The recent shift to a virtual environment allows scientists to critically reexamine how to share information with their peers and with the wider communities affected by evidence-based policy decisions. We have highlighted that in-person conferences can be inaccessible and limit diplomacy due to costs, format, and inequity. Although virtual meetings pose some accessibility challenges, 
we have identified tools that can offer a similar but more equitable experience compared to inperson meetings. We recommend that future conferences, both fully and partially virtual, consider our recommendations regarding conference attendance and talks, networking, and public engagement. These recommendations can push scientific conferences to be a more equitable, accessible space for scientists, policymakers, and the public to foster meaningful diplomatic relationships.

\section{Endnotes}

1. International Telecommunications Union. 2020. "Statistics.” International Telecommunication Union. https://www.itu.int/en/ITU-

D/Statistics/Pages/stat/default.aspx.

2. Cohen, Jason. 2020. "Data Usage Has Increased 47 Percent During COVID-19 Quarantine.” PCMag. June 5. https://www.pcmag.com/news/data-usage-has-increased47-percent-during-covid-19-quarantine.

3. Walsh, Bryan. 2020. "An infodemic of misinformation is crippling the coronavirus response ." Axios. July 8. https://www.axios.com/coronavirus-misinformationconspiracy-theories-ad2785eb-b5a2-4ea5-8158-729cb8879130.html.

4. Fleming, Nic. 2020. "Coronavirus misinformation, and how scientists can help to fight it.” Nature 583 (7814): 155-56. doi:10.1038/d41586-020-01834-3.

5. Royal Society. 2010. "New frontiers in science diplomacy." The Royal Society. January 12. https://royalsociety.org/topics-policy/publications/2010/new-frontiers-sciencediplomacy/.

6. Klemeš, Jiří Jaromír. 2016. "Scientific conferences: organisation, participation and their future." Clean Technologies and Environmental Policy 18 (2): 347-49. doi:10.1007/s10098-016-1106-2.

7. Stark, Kevin. 2020. "Conference Travel and Carbon Emissions: In the Midst of COVID19, Some People Are Doing the Math." KQED. June 19.

https://www.kqed.org/science/1966164/covid-19-is-pushing-scientific-conferencesonline-maybe-thats-where-they-belong.

8. Rowe, Nicholas E. 2019. "The Economic Cost of Attending Educational Conferences." International Journal on Social and Education Sciences 1 (1).

9. Fleming, Nic. 2020. "What's on the agenda for post-pandemic meetings?" Nature, August. doi:10.1038/d41586-020-02254-z.

10. International Congress on Catalysis. 2020. "17th International Congress on Catalysis." 2020 International Congress on Catalysis. https://2020icc.com/.

11. American Physical Society. 2020. "Quarks to Cosmos." Quarks to Cosmos 2020. https://april.aps.org/about/.

12. United Nations. 2020. "2020 UNC Ocean Conference Postponed." United Nations. https://www.un.org/sustainabledevelopment/blog/2020/04/2020-un-ocean-conferencepostponed/.

13. Sagers, Jessica. 2019. "Reimbursement policies make academia less inclusive." Science, February. doi:10.1126/science.caredit.aax0053.

14. Salomon, Dor, and Mario F Feldman. 2020. "The future of conferences, today: Are virtual conferences a viable supplement to 'live' conferences?" EMBO Reports 21 (7): e50883. doi:10.15252/embr.202050883. 
15. American Vacuum Society. 2019. “ALD/ALE 2019.” ALD 2019. https://ald2019.avs.org/register/.

16. American Vacuum Society. 2020. “ALE/ALD 2020.” ALD 2020. https://ald2020.avs.org/register/.

17. Sá, Maria José, Carlos Miguel Ferreira, and Sandro Serpa. 2019. "Virtual and Face-ToFace Academic Conferences: Comparison and Potentials." Journal of Educational and Social Research 9 (2): 35-47. doi:10.2478/jesr-2019-0011.

18. Poushter, Jacob. 2016. "Internet access grows worldwide but remains higher in advanced economies." Pew Research Center. February 22.

https://www.pewresearch.org/global/2016/02/22/internet-access-growing-worldwide-butremains-higher-in-advanced-economies/.

19. Nawaz, Sabina, and Roberta J. Cordano. 2020. "What Deaf People Can Teach Others About Virtual Communication." Harvard Business Review. August 3. https://hbr.org/2020/08/what-deaf-people-can-teach-others-about-virtual-communication.

20. Mullen, Williams. 2020. "Protecting Your Sensitive Information While Using Virtual Meeting Platforms." JDSupra. April 7. https://www.jdsupra.com/legalnews/protectingyour-sensitive-information-40102/.

21. Bosslet, Gabriel T., Hugo Carmona, Kristin M. Burkart, Jennifer McCallister, Joyce Reitzner, Maryl Kreider, and Peter H. Lenz. 2020. "Virtually Hosting a National Medical Society Conference. Lessons Learned from the 2020 Association of Pulmonary and Critical Care Medicine Program Directors Conference.” ATS Scholar, July, atsscholar.202. doi:10.34197/ats-scholar.2020-0054IN.

22. Price, Michael. 2020. "Scientists discover upsides of virtual meetings." Science 368 (6490): 457-58. doi:10.1126/science.368.6490.457.

23. MeetingPlay. 2020. "Does Virtual Networking Really Work?” MeetingPlay. https://www.meetingplay.com/blog/does-virtual-networking-really-work.

24. High Fidelity. 2020. "Live Spatial Audio and Virtual Worlds Technologies ." High Fidelity. https://www.highfidelity.com/.

25. Mozilla. 2020. "Hubs - Private social VR in your web browser." https://hubs.mozilla.com/.

26. Le, Duc Anh, Blair Maclntyre, and Jessica Outlaw. 2020. "Enhancing the experience of virtual conferences in social virtual environments." In 2020 IEEE Conference on Virtual Reality and 3D User Interfaces Abstracts and Workshops (VRW), 485-94. IEEE. doi:10.1109/VRW50115.2020.00101.

27. 3M. 2020. "State of Science Index." 3M. https://www.3m.com/3M/en_US/state-ofscience-index-survey/.

28. CAISE. 2013. Convening November 19-20 Front-End Report. Center for Research on Lifelong STEM Learning.

29. American Academy of Arts and Sciences. 2019. Encountering Science in America. The Public Face of Science Initiative. American Academy of Arts and Sciences.

30. Holmes, Ryan. 2020. "2 Million Professionals Polled On How To Make Virtual Conferences Better- Here Are Their Top 10 Hacks." Forbes. June 11. https://www.forbes.com/sites/ryanholmes/2020/06/11/i-asked-2-million-professionalshow-to-make-virtual-conferences-better--here-are-their-top-10-hacks/\#530829203132.

31. Woolston, Chris. 2020. "Learning to love virtual conferences in the coronavirus era." Nature 582 (7810): 135-36. doi:10.1038/d41586-020-01489-0. 
32. Brewer, Geoffrey. 2001. "Snakes Top List of Americans' Fears.” Gallup News. March 19. https://news.gallup.com/poll/1891/snakes-top-list-americans-fears.aspx. 